\title{
Letrozole Versus Letrozole plus Lapatinib (GW572016) in Hormone-Sensitive, HER2-Negative Operable Breast Cancer: A Double-Blind, Randomized, Phase II Study with Biomarker Evaluation (EGF109077-LAP107692/LETLOB)
}

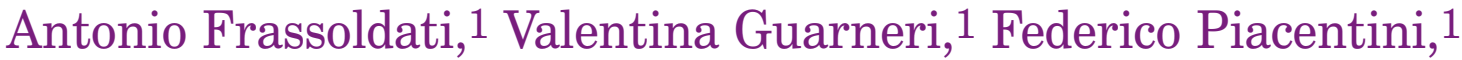 \\ Gordana Jovic, ${ }^{1}$ Simona Giovannelli, ${ }^{1}$ Cristina Oliva, ${ }^{2}$ Pier Franco Conte ${ }^{1}$
}

\begin{abstract} Many hormone receptor-positive tumors show primary or acquired resistance, possibly because of a crosstalk with other growth factor-related transduction pathways (mainly epidermal growth factor receptor family related). The LETLOB study is a European multicenter, placebo-controlled, randomized phase II trial in postmenopausal patients with hormone-sensitive, HER2-negative, stage II-IIIA (T > $2 \mathrm{~cm}, \mathrm{NO}-1$, $\mathrm{MO}$ ) breast cancer, in which letrozole or the combination of letrozole plus lapatinib will be administered for 6 months before surgery. Clinical endpoints (primary [ultrasonographic objective response], secondary [rate of pathologic complete response and of conservative surgery, safety, and time to treatment failure], and biologic [inhibition of intermediate and final biomarkers of the proliferative and apoptosis pathways and gene profile correlation with response]) will be evaluated.
\end{abstract}

Clinical Breast Cancer, Vol. 8, No. 1, 97-100, 2008

Key words: Mitogen-activated protein kinase, Multigated acquisition scan, Tyrosine kinase inhibitor

\section{Background}

More than $60 \%$ of breast cancers are hormone receptor (HR) positive, and this percentage increases in cases arising after menopause. The standard medical treatment of these tumors is represented by drugs inhibiting the estrogen-related pathways, like tamoxifen, fulvestrant, and aromatase inhibitors (AIs), or, in the premenopausal setting, by drugs suppressing ovar-

${ }^{1}$ Division of Medical Oncology, Department of Oncology and Hematology, University Hospital, University of Modena and Reggio Emilia, Modena, Italy

${ }^{2}$ Oncology Medicine Development Center, GlaxoSmithKline, Greenford, UK

Submitted: Oct 8, 2007; Accepted: Oct 9, 2007

Address for correspondence: Pier Franco Conte, MD, Department of Oncology and Hematology, University Hospital, via del Pozzo 71, 41100 Modena, Italy

Fax: 39-059-422-4429; e-mail: conte.pierfranco@unimo.it

EUDRACT number: 2006-0019707-13; NCI ClinicalTrials.gov number: NCT00422903 ian functions such as the luteinizing hormone (LH)-releasing hormone agonists. Unfortunately, half of the patients show de novo resistance to these drugs, and almost all cases eventually develop secondary or acquired resistance. ${ }^{1}$ Many studies have suggested that the crosstalk between estrogen-related gene activation and growth factor-related transduction pathways plays an important role in the occurrence of resistance 2,3 ; this could clearly be the case in HR-positive/HER2-positive cancers. ${ }^{4}$ However, simultaneous positivity for HR and HER2 is observed in a minority of cases, although resistance arises mainly in HR-positive/HER2-negative tumors.

In fact, the so-called HER2-negative cancers comprise tumors with complete absence of HER2 receptors and tumors with low or moderate expression (IIC score 1 and 2). Moreover, even score 0 HER2 cells probably possess very low expression of HER2 receptors because HER2 is essential in the control of normal cell growth. Finally, other epidermal growth factor (EGF)-family receptors (EGFR, HER3, and HER4), or insulin-like growth factor-1 (IGF-1) receptor might

Electronic forwarding or copying is a violation of US and International Copyright Laws.

Authorization to photocopy items for internal or personal use, or the internal or personal use of specific clients, is granted by CIG Media Group, LP,

ISSN \#1526-8209, provided the appropriate fee is paid directly to Copyright Clearance Center, 222 Rosewood Drive, Danvers, MA 01923 USA 978-750-8400. 
be expressed in estrogen receptor (ER)-positive breast cancer cells. The classical genomic action of estrogen occurs through an interaction between the ER complex (with the cooperation of coactivator and corepressor proteins) with the estrogen response elements of target genes. ${ }^{5}$ An alternative, nonclassical pathway consists of the activation of other promoter regions, like AP-1, by transcriptional factors (as Jun and Fos) coupled with an ER-coactivator complex functioning as costimulator agent. The intracellular level of coactivators and corepressors, regulated by many different pathways, would be responsible for the net effect of the ER-dependent pathway. 6 In contrast with this nuclear-initiated steroid signalling, a more recently identified membrane-initiated steroid signalling can be active as a nongenomic pathway.7,8 This membrane ER can activate, directly and indirectly, other membrane tyrosine kinases (TKs; related to EGFR, HER2-receptor, IGF1-R), which in turn can activate downstream pathway messengers like mitogen-activated protein kinase (MAPK), PI3K, and AKT. 8

These systems show a bidirectional crosstalk, creating a complex network aimed at cell survival when one of them is downregulated or suppressed. A compensating action of EGFR/HER2- and IGF1-R-related pathways has been observed in long-term estrogen-depleted (LTED) breast cancer cells and has been considered responsible for primary and acquired resistance to AIs. ${ }^{9}$ A large amount of preclinical and clinical evidence supports this opinion.

Overexpression of EGFR/HER2 enhances both genomic and nongenomic ER action, inducing tamoxifen resistance10; MCF7 cells transfected with the HER2 gene develop tamoxifen-dependent growth11; LTED breast cancer cells exhibit enhanced levels of HER2, EGFR, and MAPK. ${ }^{1}$ One interesting observation is that ER-positive cells becoming resistant to tamoxifen continue to express ER and can maintain an ER-directed therapy sensitivity, indicating that ER and EGFR are dynamically involved in the process, which can lead to different driving pathways in different situations.12 In fact, in MCF7 cells exposed to tamoxifen in the long term, the sensitivity to selective ER modulators can be restored with drugs targeting EGFR (like gefitinib) or HER2 (like trastuzumab or pertuzumab). 13

In clinical studies of primary therapy, ER-positive/HER2positive cancer shows lower sensitivity to tamoxifen, while AIs show activity independent of HER2 status. ${ }^{14}$ These observations have been confirmed by retrospective analyses of large adjuvant trials comparing tamoxifen with AIs. ${ }^{15,16}$

Two neoadjuvant trials investigated the combination of an AI with gefitinib, an inhibitor of the TK of EGFR.17,18 The combination showed higher activity when patients were selected on the basis of EGFR positivity, while no apparent effect was observed in negative cases. However, in a preclinical and clinical model, the combination of tamoxifen and gefitinib significantly delayed the onset of hormone resistance in ERpositive/HER2-positive breast cancer. ${ }^{19,20}$

The combination of anastrozole and trastuzumab has been investigated in a phase III randomized trial of 208 patients with ER- and HER2-positive advanced breast cancer. ${ }^{21}$ Patients treated with the combined therapy showed longer progression-free survival (4.8 months vs. 2.4 months) and a higher response rate ( $20.3 \%$ vs. $6.8 \%$ ) was observed among the 147 evaluable patients.

Lapatinib is an orally active small molecule that inhibits the EGFR and HER2 TKs. ${ }^{22}$ One of the advantages of this compound is the simultaneous blockade of multiple pathways dependent from EGFR and HER2 that could replicate the inhibition observed in vitro with the combination of gefitinib, trastuzumab, pertuzumab, and tamoxifen. The most attractive result of the combination of hormone therapy and lapatinib is the potential for a delay of the tumor growth, conceivably related to the lack of activation of the EGFR and HER2 pathways after the blockade of the hormonal pathway. A cooperation of tamoxifen and lapatinib has already been observed by $\mathrm{Chu}$ et al with enhanced cell-cycle arrest and reduced ER-dependent gene transcription.23 In advanced disease, a trial exploring the combination of letrozole with lapatinib (EGF30008) in ER-positive tumors (irrespective of HER2 status) recently completed accrual. However, many of these patients have already been treated with tamoxifen, and no trial has been conducted in naive patients.

On these premises, we have designed a preoperative trial combining an AI (letrozole) with a dual HER1 (EGFR) and HER2 TK inhibitor (lapatinib) in postmenopausal patients with HR-positive/HER2-negative breast cancer.

\section{The EGF109077-LAP107692/LETLOB Trial}

Until recently, the majority of the data have been limited to cytotoxic chemotherapy, but preoperative endocrine therapy is becoming an attractive alternative in cases of hormone sensitive tumors. In fact, the majority of preoperative chemotherapy trials have shown that the probability of obtaining a complete response (CR) to chemotherapy is significantly lower in cases of hormone-sensitive tumors, thus suggesting a major role for endocrine manipulation for these patients. ${ }^{24}$

In hormone-sensitive breast cancer, primary treatment with AIs resulted in higher clinical response rates and conservative surgery rates than with tamoxifen.18 Clinical objective responses (cORs) to anastrozole and to letrozole administered for 3 months have been obtained in about $25 \%$ to $60 \%$ of the patients, with very few pathologic complete remissions (about 3\%-5\%).

The LETLOB trial is the first study conducted in a neoadjuvant setting in which a combination of letrozole and lapatinib is administered to postmenopausal women with HR-positive/HER2-negative breast cancer.

\section{Endpoints of the Trial}

The primary endpoint of LETLOB is to estimate the percentage of cORs (complete plus partial measured by ultrasonogram) in the breast. The secondary endpoints include: (1) estimate the percentage of pathologic CRs in the breast and axillary nodes; (2) estimate the percentage of conservative surgery; (3) assess the safety profile of the combination of hormonal treatment plus lapatinib; (4) evaluate the time 
to treatment failure from the start of primary therapy; (5) evaluate the percentage of inhibition of intermediate and final biomarkers of the proliferative and the apoptosis pathways induced by the hormonal treatment plus lapatinib or placebo; and (6) evaluate the association between tumor gene expression at diagnosis and pathologic response in the 2 treatment arms.

\section{Inclusion and Exclusion Criteria}

Eligible patients must have histologically confirmed infiltrating primary breast cancer of $>2 \mathrm{~cm}$ in largest clinical diameter. Tumors must be ER and/or progesterone receptor positive ( $>10 \%$ of positive cancer cell assessed by immunohistochemistry [IHC]), and HER2 negative (IHC score 0-2+ or florescence in situ hybridization negative).

The trial is open to postmenopausal patients with Eastern Cooperative Oncology Group performance status 0-1 (defined by $\geq 1$ of the following: [1] aged $\geq 60$ years; [2] aged $<60$ years and amenorrheic for $\geq 12$ months before day 1 ; [3] aged $<60$ years and amenorrheic for $<12$ months before day 1 without a uterus or with LH and follicle stimulating hormone values within postmenopausal range; [4] previous bilateral oophorectomy; and/or [5] previous radiation castration with amenorrhea for $\geq 6$ months).

A normal cardiac function, with left ventricular ejection fraction (LVEF) within the institutional range of normal as measured by echocardiogram or multigated acquisition (MUGA) scan is a prerequisite for enrollment in the study. Other inclusion criteria are the presence of normal organ and marrow function, the possibility to swallow and retain oral medication, and the ability to understand and the willingness to sign a written informed consent document.

On the contrary, patients will be excluded if they have stage IIIB, IIIC, inflammatory, or stage IV breast cancer; if they have received previous treatment with chemotherapy, endocrine therapy or radiotherapy, or previous treatment with EGFR-targeting therapies; or if they receive concomitant medication classified as CYP3A4 inducers or inhibitors.

\section{Sample Size}

The sample size has been estimated by using the 2 -stage Simon's design. We will consider interesting the combination of letrozole plus lapatinib if the incidence of cOR will be equal to $70 \%$ (a $40 \%$ increase compared with the cOR expected with hormonal therapy alone). With $\alpha=10 \%$ and $\beta=10 \%$, the following sample sizes have been obtained: (1) arm A: letrozole plus placebo; first stage; 22 patients. If $\geq 8$ responses are recorded in the first 22 patients, 24 additional patients will be treated, for a total of 46 patients. (2) Arm B: letrozole plus lapatinib; first stage; 21 patients. If $\geq 12$ responses are recorded in the first 21 patients, 24 additional patients will be treated, for a total of 45 patients.

\section{Trial Design}

LETLOB is a European, multicenter, placebo-controlled, randomized phase II trial in postmenopausal patients with hormone-sensitive, HER2-negative stage II-IIIA breast cancer ( $\mathrm{T}>2 \mathrm{~cm}, \mathrm{~N} 0-1, \mathrm{M} 0)$.

\section{Figure 1 LETLOB Trial Design}

Study Plan

Postmenopausal patients with breast cancer $(T>2 \mathrm{~cm})$ Stage II-IIIA, HR+, HER2-

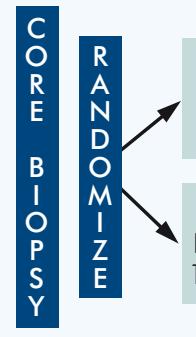

Letrozole $2.5 \mathrm{mg}$ plus placebo orally daily for 6 months

Arm B:

Letrozole $2.5 \mathrm{mg}$ plus Lapatinib

$1500 \mathrm{mg}$ orally daily for 6 months

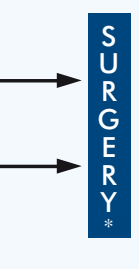

*Surgery within 2 weeks after the last letrozole or lapatinib/placebo dose.

After core biopsy of the primary breast nodule, patients will receive oral letrozole $2.5 \mathrm{mg}$ plus oral lapatinib $1500 \mathrm{mg}$ daily or placebo for 6 months before surgery (Figure 1).

\section{Monitoring of Cardiac Safety}

Only patients with LVEF within the institutional limits of normal at baseline as measured by echocardiography or MUGA scan are eligible for this protocol. Left ventricular ejection fraction evaluation is repeated after 12 weeks and at the completion of treatment. More frequent assessment of LVEF should be repeated in any patients with signs or symptoms suspicious of cardiac dysfunction.

\section{Biomarker Evaluation and Gene Expression Profile}

To define the effects of treatment on the downstream pathways of the EGFR family, the following biomarkers are centrally evaluated: (1) EGFR, HER2; (2) pTEN, pAKT, pMAPK; (3) apoptosis (terminal uridine nick-end labeling test); and (4) Ki-67. These parameters are evaluated by IHC, on paraffin-embedded specimens obtained from diagnostic core biopsy of the primary lesion, and from surgical specimens. Moreover, fresh tumor tissue from the diagnostic core biopsy is collected and snap frozen to perform a microarray analysis of the gene expression profile before treatment and to evaluate its correlation with response.

\section{Conclusion}

The LETLOB study represents the first in vivo evaluation of the activity of the combination of an AI with lapatinib in patients naive to ER-positive/HER2-negative breast cancer. The study design will help in understanding the mechanism regulating the onset of hormone resistance at clinical and biologic levels. The combination of different agents able to induce a contemporary blockade of different metabolic pathways regulating proliferation and apoptosis is one of the areas of main interest in order to gain a more powerful targeted therapy. 25 


\section{References}

1. Massarweh S, Schiff R. Resistance to endocrine therapy in breast cancer: exploiting estrogen receptor/growth factor signaling crosstalk. Endocr Relat Cancer 2006; 13(suppl 1):s15-24.

2. Schiff R, Massarweh SA, Shou J, et al. Advanced concepts in estrogen receptor biology and breast cancer endocrine resistance: implicated role of growth factor signaling and estrogen receptor coregulators. Cancer Chemother Pharmacol 2005; 56(suppl 1):10-20.

3. Massarweh S, Osborne CK, Jiang S, et al. Mechanisms of tumour regression and resistance to estrogen deprivation and fulvestrant in a model of estrogen receptor-positive, HER-2/neu-positive breast cancer. Cancer Res 2006; 66:8266-73.

4. Pietras RJ, Arboleda J, Reese DM, et al. HER-2 tyrosine kinase pathway targets estrogen receptor and promotes hormone-independent growth in human breast cancer cells. Oncogene 1995; 10:2435-46.

5. Osborne CK, Schiff R. Estrogen-receptor biology: continuing progress and therapeutic implications. J Clin Oncol 2005; 23:1616-22.

6. Osborne CK, Bardou V, Hopp TA, et al. Role of the estrogen receptor coactivator AIB1 (SRC-3) and HER-2/neu in tamoxifen resistance in breast cancer. J Natl Cancer Inst 2003; 95:353-61.

7. Chung Y-L, Sheu M-L, Yang S-C, et al. Resistance to tamoxifeninduced apoptosis is associated with direct interaction between Her2/neu and cell membrane estrogen receptor in breast cancer. Int $J$ Cancer 2002; 97:306-12.

8. Nemere I, Pietras RJ, Blackmore PF. Membrane receptors for steroid hormones: signal transduction and physiological significance. $J$ Cell Biochem 2003; 88:438-45.

9. Santen RJ, Song RX, Zhang Z, et al. Long-term estradiol deprivation in breast cancer cells up-regulates growth factor signaling and enhances estrogen sensitivity. Endocr Relat Cancer 2005; 12(suppl 1):s61-73.

10. Hutcheson IR, Knowlden JM, Madden T-A, et al. Oestrogen receptor-mediated modulation of the EGFR/MAPK pathway in tamoxifenresistant MCF-7 cells. Breast Cancer Res Treat 2003; 81:81-93.

11. Gutierrez MC, Detre S, Johnston S, et al. Molecular changes in tamoxifen-resistant breast cancer: relationship between estrogen receptor, HER-2, and p38 mitogen-activated protein kinase. J Clin Oncol 2005; 23:2469-76.

12. Arpino G, Weiss $\mathrm{H}$, Wakeling A, et al. Complete disappearance of ERC/HER2C breast cancer xenografts with the combination of gefitinib, trastuzumab, and pertuzumab to block HER2 cross-talk with ER and restore tamoxifen inhibition. Breat Cancer Res Treat 2004; 88(suppl 1):s15 (Abstract 23).

13. Arpino G, Weiss H, Lee AV, et al. Estrogen receptor-positive, progesterone receptor-negative breast cancer: association with growth factor receptor expression and tamoxifen resistance. $J$ Natl Cancer Inst 2005; 97:1254-61.

14. Ellis MJ, Coop A, Singh B, et al. Letrozole is more effective neoadjuvant endocrine therapy than tamoxifen for ErbB-1- and/or ErbB-2-positive, estrogen receptor-positive primary breast cancer: evidence from a phase III randomized trial. J Clin Oncol 2001; 19:3808-16.

15. Viale G, Regan MM, Maiorano E, et al. Prognostic and predictive value of centrally reviewed expression of estrogen and progesterone receptors in a randomized trial comparing letrozole and tamoxifen adjuvant therapy for postmenopausal early breast cancer: BIG 1-98. $J$ Clin Oncol 2007; 25:3846-52.

16. Dowsett M, Allred C, Knox J, et al. Relationship between quantitative estrogen and progesterone receptor expression and human epidemal growth factor receptor (HER-2) status with recurrence in the Armidex, Tamoxifen, Alone or in Combination Trial. $J$ Clin Oncol 2008; [e-pub ahead of print].

17. Eiermann W, Paepke S, Appfelstaedt J, et al. Preoperative treatment of postmenopausal breast cancer patients with letrozole: a randomized double-blind multicenter study. Ann Oncol 2001; 12:1527-32.

18. Smith IE, Dowsett M, Ebbs SR, et al. Neoadjuvant treatment of postmenopausal breast cancer with anastrozole, tamoxifen or both in combination: the Immediate Preoperative Anastrozole, Tamoxifen, or Combined with Tamoxifen (IMPACT) multicentre double-blind randomized trial. J Clin Oncol 2005; 23:5108-16.

19. Polychronis A, Sinnett HD, Hadjiminas D, et al. Preoperative gefitinib versus gefitinib and anastrozole in postmenopausal patients with oestrogen-receptor positive and epidermal-growth-factorreceptor-positive primary breast cancer: a double-blind placebocontrolled phase II randomised trial. Lancet Oncol 2005; 6:383-91.

20. Robertson JF, Gutteridge E, Cheung KL, et al. Gefitinib (ZD1839) is active in acquired tamoxifen-resistant oestrogen receptor (ER)positive and ER negative breast cancer: results from a phase II study. Proc Am Soc Clin Oncol 2003; 22:7 (Abstract 23).

21. Mackey JR, Kaufman B, Clemens M, et al. Trastuzumab prolongs progression-free survival in hormone-dependent and HER2-positive metastatic breast Cancer. Breast Cancer Res Treat 2006; 100(suppl 1):s5 (Abstract 3).

22. Xia W, Mullin RJ, Keith BR, et al. Anti-tumor activity of GW572016: a dual tyrosine kinase inhibitor blocks EGF activation of EGFR/ erB2 and downstream Erk1/2 and AKT pathways. Oncogene 2002; 21:6255-63.

23. Chu I, Blackwell K, Chen S, et al. The dual ErbB1/ErbB2 inhibitor, lapatinib (GW572016), cooperates with tamoxifen to inhibit both cell proliferation- and estrogen-dependent gene expression in antiestrogen-resistant breast cancer. Cancer Res 2005; 65:18-25.

24. Semiglazov VF, Ivanov VG, Semiglazov VV, et al. Neoadjuvant endocrine therapy vs chemotherapy for postmenopausal ER-positive breast cancer patients. Breast Cancer Res Treat 2004: 88(suppl 1):s106 (Abstract 2090).

25. Johnston SR, Head J, Pancholi S, et al. Integration of signal transduction inhibitors with endocrine therapy: an approach to overcoming hormone resistance in breast cancer. Clin Cancer Res 2003; $9: 524 \mathrm{~s}-32 \mathrm{~s}$ 